\title{
Análisis mediante simulación numérica de la inyección de baches surfactante/polímero para el campo yariguí-cantagallo operado por Ecopetrol S.A.
}

\author{
pags $21-41$ \\ Grupo de Investigación: Simulación de yacimientos y Recobro Mejorado del Petróleo \\ Línea de investigación: Simulación de yacimientos

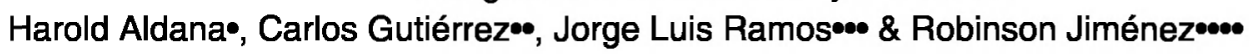

Recibido: 27 de mayo de 2015

\section{RESUMEN}

El Campo Yariguí-Cantagallo fue descubierto a mediados de los años 40's, y adquirido por ECOPETROL S.A en el año de 1975. En el mes de Noviembre de 2008, y debido a la disminución de la presión del yacimiento, Ecopetrol decide intervenirlo iniciando la implementación de métodos de recobro secundario. Se realizaría mediante la incorporación de un proceso de inyección de agua, con el fin de mantener la presión del yacimiento y que la producción de hidrocarburos aumente considerablemente. En el año 2010 el Instituto Colombiano del Petróleo de ECOPETROL S.A. inicia una evaluación de la viabilidad del recobro mejorado mediante inyección de químicos en el campo, con el fin de incrementar la producción de petróleo y el factor de recobro. A su vez, disminuir la producción de agua en los pozos productores.

Dicha evaluación será hecha mediante el uso de la simulación numérica de yacimientos. Los beneficios del proceso EOR serán analizados a partir de la producción de agua y petróleo, así como de los posibles beneficios económicos que este puede generar.

Palabras Clave: Simulación Numérica, Recobro Mejorado, Inyección Química, Baches Surfactante/Polímero, Incremental Producción.
Aceptado: 12 de junio de 2015

\section{ABSTRACT}

Yarigui-Cantagallo Field was discovered in the mid40's, and acquired in 1975 by Ecopetrol SA. In the month of November 2008, and due to reduced reservoir pressure, Ecopetrol decided to do an intervention, beginning by to implement secondary recovery methods. It would be made by adding water injection wells in order to maintain reservoir pressure. Is expected the fact that oil production to increase considerably. Ecopetrol began a process of evaluating the feasibility of recovery in the tertiary (chemical injection) field, in order to increase oil production. In turn, to reduce water production in producing wells.

Such assessment shall be made using the numerical reservoir simulation. The benefits of EOR process will be analyzed from the water and oil production as well as the potential economic benefits it can generate.

Keywords: Numerical Simulation, Enhanced Oil Recovery, Chemical Injection, Surfactant/Polymer Slug, Incremental Production.

\footnotetext{
- Estudiante coinvestigador. Fundación Universidad de América Ing. Petróleos.

- Estudiante coinvestigador. Ing. Petróleos Fundación Universidad de América

... Docente investigador. Ing. Petróleos Fundación Universidad de América. Jorge Ramos@investigadores.uamerica.edu.co

•..• Ingeniero de petróleos, Convenio ICP-Ecopetrol S.A.
} 


\section{INTRODUCCIÓN}

El Campo Yariguí-Cantagallo está ubicado en la Cuenca del Valle Medio del Madalena, en el departamento de Santander, Colombia, al sur del cauce del Río Magdalena. El campo ha tenido una trayectoria de explotación de petróleo desde los años 40's, hasta la actualidad, involucrando una disminución de presión considerable del yacimiento. Seguido a esto, reduciendo la tasa de producción diaria de petróleo, hasta a $\mathbf{5 0 0 0}$ BOPD. Debido a ello y al aumento considerable del corte de agua, Ecopetrol S.A. inició la implementación de recobro secundario en el campo, iniciando una campaña de inyección de agua a partir de Noviembre del 2008, con el fin de aumentar la tasa de recuperación de petróleo.

De esta manera, se evaluó y analizó la viabilidad técnica de inyección de químicos en el campo, mediante simulación numérica, describiendo las propiedades más importantes del yacimiento. De esta manera, aplicar un análisis de sensibilidades, permitiendo describir el escenario más adecuado y pronosticar detalladamente la producción de petróleo del campo.

De acuerdo a lo expuesto anteriormente y a la creciente demanda energética, se abre una ventana de oportunidad a la investigación de diferentes métodos. Estos contribuirian en el aprovechamiento eficiente de la energía del yacimiento, así mismo, a la mejora del desplazamiento del petróleo en el reservorio. Este se resume finalmente en el aumento en la recuperación de petróleo, incremento en las ganancias y la recuperación rápida del capital invertido. Contribuye así al desarrollo tecnológico de Ecopetrol S.A., además de generar desarrollo en el país aumentando el factor de recobro promedio nacional.

\section{Recobro mejorado asistido por químicos}

El recobro mejorado de petróleo, asistido por químicos, ha sido usado ampliamente en desarrollos alrededor del mundo, maximizando la recuperación del petróleo. Fue considerada su- ficiente la implementación de procesos de recobro, con la adición de un solo químico (surfactante o polímero) en las primeras etapas. Sin embargo, proyectos piloto demostraron la superioridad de combinar el desplazamiento de surfactante con el polimero. La solución polimérica mejoraba la eficiencia de barrido macroscópica, a la vez que el surfactante aumentaba la eficiencia de barrido microscópica, creando mejores condiciones de desplazamiento para el petróleo, y aunado al hecho, una mayor recuperación de petróleo. Ecopetrol, en su plan de desarrollo de campos maduros, inició un ambicioso plan que considera la investigación de tecnologías, para maximizar el factor de recobro, y así mismo, aumentar la vida productiva de los yacimientos maduros. Una de estas tecnologías podría ser la inyección de baches surfactante-polímero. La inyección de baches surfactante-polímero es una modificación de la tecnología SP convencional, la cual considera las siguientes etapas de inyección:

- Pre-flujo (Opcional). Es un bache de agua generalmente, que tiene como función ajustar el pH y la salinidad de la salmuera (desplazando iones de sodio, calcio y magnesio), con esto se busca evitar cualquier reacción química entre la solución inyectada y los fluidos de la formación. Sin embargo, la solución micelar se desarrolla para tolerar la salmuera del yacimiento. En ocasiones, este bache tiene una baja concentración de un adsorbente de sacrificio y tiene como finalidad minimizar las pérdidas por adsorción en el proceso posterior. Esto se logra haciendo que la cantidad del compuesto usado sea adsorbido por los granos de la roca.

- Solución micelar. Este bache se caracteriza por tener una baja tensión interfacial con el petróleo, facilitando la movilidad de este, dando paso a la formación de un banco de petróleo delante del bache. Por lo general, cuando se aplica este tipo de procesos la saturación de petróleo en el yacimiento es residual y la producción de petróleo no llega hasta que el banco de petróleo irrumpe en los pozos productores. 
- Regulador de movilidad. Este bache es una solución polimérica que busca crear una relación de movilidad favorable para la solución micelar, asegurando un desplazamiento uniforme y maximizando la eficiencia de barrido volumétrico. A medida que se acerca al pozo inyector, a la solución polimérica se le disminuye la concentración de polímeros para finalmente llegar a un bache de agua de inyección.

- Fluido desplazante. Corresponde a la inyección continua de agua que se encarga de desplazar todos los baches mencionados anteriormente.

La inyección de baches surfactante-polímero considera el mecanismo de desplazamiento descrito anteriormente, sin embargo, este se repetirá cuantas veces resulte ser económicamente viable. La Figura 1 esquematiza una inyección de dos baches surfactante-polímero.

Figura 1 Esquema de inyección de Baches surfactante/polímero

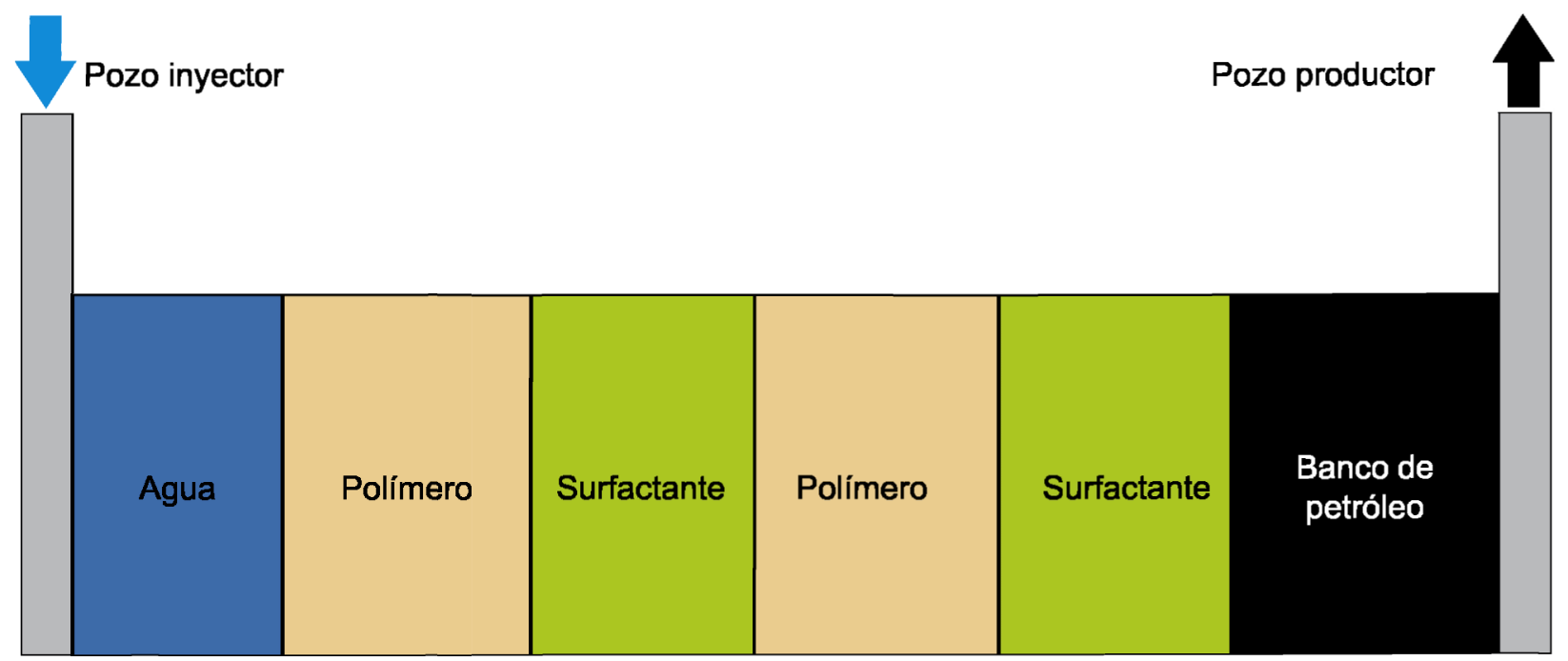

Fuente: Los Autores.

\section{MÉTODO}

\section{Químicos usados en el proceso de baches SP}

Para analizar la respuesta del yacimiento a la inyección de baches SP, se seleccionó una poliacrilamida parcialmente hidrolizada (HPAM) y un surfactante no iónico. Las propiedades de dichos componentes se encuentran adscritas en la Tabla 1 y la Tabla 2 respectivamente.

\section{Permeabilidades relativas y tensión interfacial}

Siendo la inyección de baches SP, un proceso asociado a la interacción de agentes químicos, con el sistema roca/fluido; la acción del surfactante es un punto clave en la construcción del modelo de simulación, en la tensión interfacial y la variación de los endpoints. Sin embargo, el surfactante seleccionado en este desarrollo no ha sido probado en núcleos 
del Campo Yariguí-Cantagallo. Por ello, Rao \& Ayirata, (2006) son antecedentes del uso de surfactantes no-iónicos en el desplazamiento de petróleo, y sirvieron como base en la construcción y modelamiento de las permeabilidades relativas. También, en la tensión interfacial para el Campo Yarigui-Cantagallo. Los resultados de laboratorio reportados por Rao \& Ayirata (Ibídem) se pueden observar en la Tabla 3.

Sin embargo, las permeabilidades relativas, tanto de petróleo como agua, no siguen una tendencia definida, y por lo tanto, se requiere hacer un ajuste matemático. Por otra parte, los datos presentados con anterioridad, no podían describir el comportamiento de los fluidos presentes en el yacimiento. Esto, porque las permeabilidades relativas y las saturaciones diferían de las presentes, en los núcleos usados en laboratorio. Por dicha razón, se desarrollaron modelos para ajustar las variaciones conseguidas por el surfactante, con las propiedades presentes en el yacimiento (Aldana \& Gutiérrez, 2015). Para la saturación de agua irreducible, se obtuvo el modelo planteado en la Ecuación 1:

$S w_{i r r}=\alpha+\frac{\theta^{*} C s^{\eta}}{\kappa^{\eta}+C s^{\eta}}$

Donde:

Swirr = Saturación de agua irreducible. (Fracción),

$\mathrm{a}=4.05 \mathrm{E}-01$,

$\theta=2.02 \mathrm{E}+02$,

$\eta \quad=6.06 \mathrm{E}-01$,

$\mathrm{K}=2.42 \mathrm{E}+08$

Cs = Concentración del surfactante (ppm).

A partir del modelo descrito previamente, se recalcularon los datos de la Tabla 3, para poder modelar la propiedad evaluada, como la saturación irreducible de agua. Con los datos recalculados, se procedió a hacer una normalización (Ecuación 2), que estableció una relación directa, entre los resultados obtenidos en laboratorio, y los valores base del Campo (Tabla 4).
Swirr,norm $=\frac{\text { Swirr }}{\text { swirr@Cs }=0 p p m}$

Hecha la normalización, se procede a determinar los valores para la saturación irreducible de agua, a diferentes concentraciones de surfactante, aplicables al Campo. Para la determinación del cambio de la saturación residual de petróleo, y de las permeabilidades relativas de agua. Se siguió, de manera análoga, el proceso de determinación de los valores de saturación de agua irreducible,

En cuanto a la saturación residual de petróleo, se modeló a partir de la Ecuación 3.

$S O_{r}=\frac{a b+c^{*} C s^{d}}{b+C s^{d}}$

Donde:

Sor = Saturación de petróleo residual (Fracción),

a $=2.90 \mathrm{E}-01$,

$\mathrm{b}=2.31 \mathrm{E}+05$,

c $=2.18 \mathrm{E}-02$,

$\mathrm{d}=1.85 \mathrm{E}+00$,

Cs es la concentración del surfactante (ppm).

La permeabilidad relativa del petróleo mantendrá un valor constante de 1.0, pues este es el valor inicial del Campo, previo a la inyección de surfactantes. Se parte de la premisa de la mejora de movilidad gracias al surfactante, pero dicho valor no variará con la aplicación del mismo. Por último, la permeabilidad relativa del agua fue modelada a partir de la regresión lineal mostrada en la Ecuación 4.

$K r w=2 E-05(C s)+0.0709$

Donde:

Krw = Permeabilidad relativa del agua (Fracción) y

Cs es la concentración del surfactante (ppm). 
Un resumen de los modelos previamente descritos, asi como la variación de la tensión interfacial, con la concentración de surfactante, puede visualizarse en la Figura 2. De igual for- ma, los valores de permeabilidades relativas y saturaciones irreducibles, de los fluidos usados para la construcción del modelo de simulación, se encuentran anexas en la Tabla 5.

Figura 2. Modelos de ajuste.
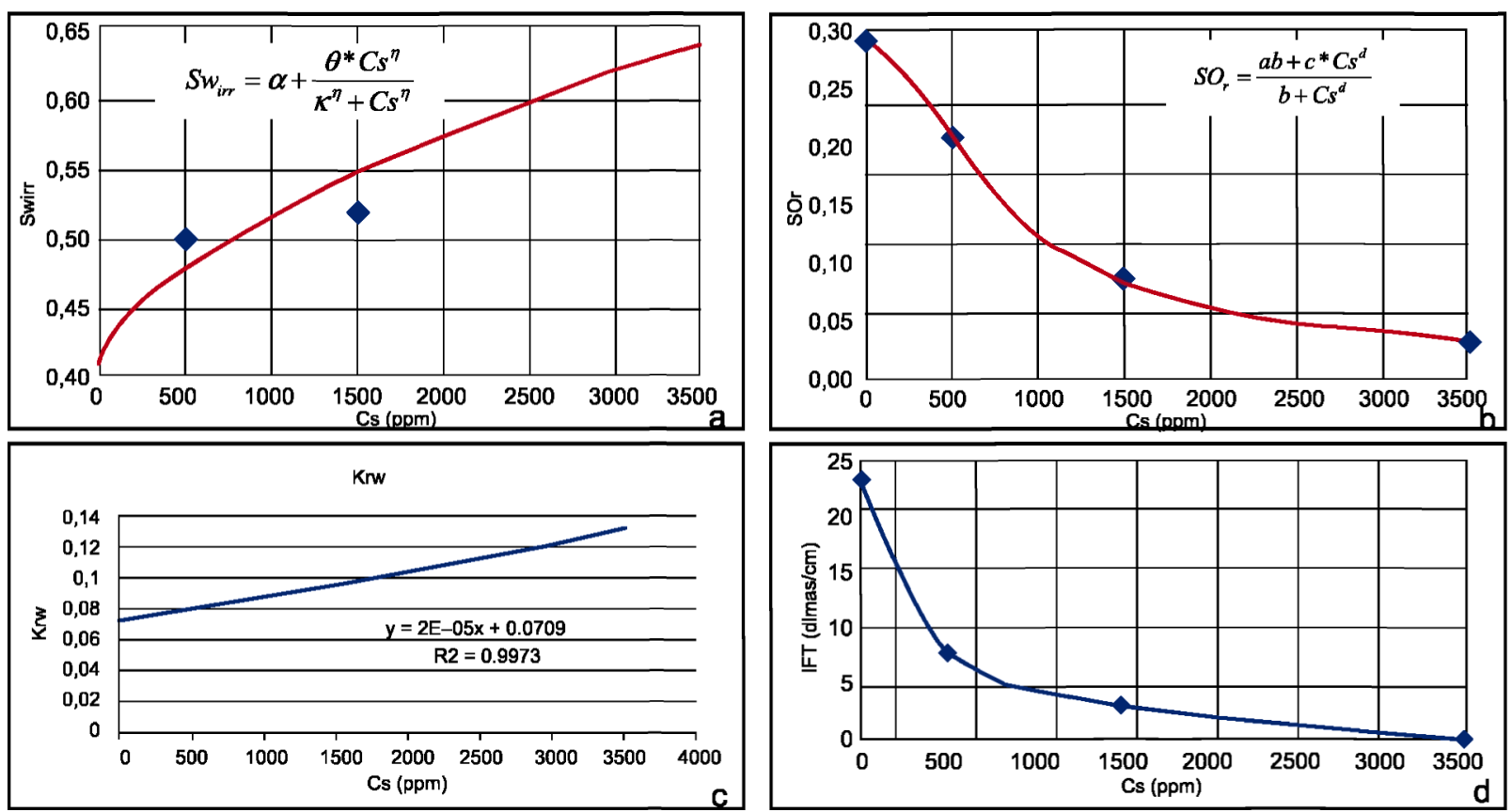

Fuente: Los Autores.

a) Saturación de agua irreducible vs. Concentración del surfactante (ppm),

b) Saturación de petróleo residual vs. Concentración del surfactante (ppm), Permeabilidad relativa del agua vs. Concentración del surfactante (ppm) y d) Tensión interfacial (dinas/cm) vs. Concentración del surfactante (ppm).

\section{Condiciones operativas}

Para la evaluación de la recuperación incremental de petróleo conseguida con la inyección de baches SP, se consideró hacer una evaluación comparativa con la producción asociada a un recobro secundario (Inyección de agua) y dos procesos de recobro mejorado asistidos por químicos (Inyección de polímeros e Inyección convencional de surfactante/polímero) en un periodo de evaluación de 10 años. Adicional a esto, los escenarios están configurados a una tasa de inyección de 1500 BFPD y una presión máxima de inyección de 4200 psi.
Se tienen los resultados del proceso de recuperación secundaria (inyección de agua), una inyección hipotética de polímero y una inyección hipotética SP convencional.

Análisis de sensibilidades

Con el objetivo de encontrar el efecto de diferentes parámetros operativos (Tasa de inyección, número de baches, concentración de los químicos, distribución de los químicos en el bache y tamaño del bache) se idearon diferentes escenarios, para obtener los parámetros adecuados como respuesta al proceso de inyección de baches SP. Se definió un escenario 
base (Escenario 1) el cual servirá como punto de referencia para la evaluación de los escenarios construidos del escenario de sensibilidad. Las propiedades de este escenario se describen en la Tabla 6.

\section{Evaluación}

Dicha evaluación considera la viabilidad de la inyección, de baches surfactante/polímero en el reservorio.

\section{Número de baches}

Para este escenario se configuraron tres escenarios, los cuales consideran dos, tres y cuatro baches SP. El escenario que concibe la inyección de dos baches, será considerado como escenario base. Servirá de referencia para las demás variables objeto de estudio. La recuperación asociada al número de baches resultó mayor cuando los baches inyectados fueron dos. Esto se asocia, a la cantidad de agua que es inyectada, dadas unas condiciones operativas iguales en todos los escenarios, y luego de la invasión química. Los resultados de esta sensibilización están asociados a la Tabla 7 y Gráfica 1.

\section{Distribución del bache}

Se diseñaron tres escenarios que consideraban la distribución de los químicos en el bache. Es decir, la cantidad de surfactante -en fracciónde cada químico respecto al tamaño total del bache (5\%). El primer escenario es el escenario base. El segundo (Escenario 4) contempla una distribución del $50 \%$ del volumen de cada bache, para cada químico. Por último, el tercer escenario (Escenario 5) considera que la cantidad de surfactante sea el $25 \%$ del tamaño total de cada bache, y el restante $75 \%$ será para la solución polimérica. Los resultados de esta sensibilización están asociados en la Tabla 8 y Gráfica 2.
Tasa de inyección

Este estudio contempla la incidencia de la tasa de inyección, en la recuperación de petróleo, asociada a la inyección de baches SP. Para este análisis se consideran tres escenarios: el caso base, el Escenario \# 6, y el Escenario \# 7, los cuales contemplan unas tasas de inyección de 2000 y 1000 BFPD. Los resultados de este análisis de sensibilidad se pueden visualizar a través de la Tabla 9 y la Gráfica 3.

Tamaño del bache

Este estudio contempla la incidencia del tamaño de los baches inyectados. Para esto se construyeron tan solo dos escenarios: el caso base y el Escenario 8. Éste contempla la inyección de baches SP, con un tamaño del $1.75 \%$ del VP. Los resultados para este estudio se resumen en la Tabla 10 y la Gráfica 4

\section{Concentración del surfactante}

Este estudio contempla la incidencia de la concentración de surfactante en la recuperación de petróleo. Para ello se contemplan cuatro escenarios. El caso base, será considerado junto a los Escenarios 9, 10 y 11. Estos escenarios consideran concentración del surfactante de 2000,2500 y 3000 ppm, respectivamente. Los resultados de este análisis de sensibilidad se pueden visualizar a través de la Tabla 11 y la Gráfica 5.

\section{Concentración del polímero}

Este estudio considera la incidencia de la variación de la concentración de petróleo, sobre la recuperación del mismo. En este análisis se contemplan cuatro escenarios. Estos son: Caso base, Escenario 12, 13 y 14. Dichos escenarios consideran una concentración de polímero de $1500,1000,2000$ y 2500 ppm, respectivamente. Los resultados de ese análisis se encuentran anexos en la Tabla 12 y la Gráfica 6. 
Disminución gradual de los químicos inyectados.

En este caso se consideraron dos escenarios, el caso base y el Escenario 15 el cual considera dos baches SP, con las siguientes características:

- Bache \# 1. Concentración de surfactante de 3500 ppm y concentración de polímero 1500 ppm.

- Bache \# 2. Concentración de surfactante de 2000 ppm y concentración de polímero 1000 ppm.

De esta manera se considera una disminución gradual de los químicos a medida que los baches SP son inyectados. Los resultados de este análisis se encuentran resumidos en la Tabla 13 y la Gráfica 7 .

Concentración equivalente de los químicos

Con el objetivo de disminuir la incertidumbre asociada a la concentración de los químicos, se crearon escenarios que consideran una con ambos químicos se manejaran en las mismas concentraciones. Por ejemplo, si el surfactante se inyecta a una concentración de 2000 ppm, el polímero será inyectado a esa misma concentración. Los escenarios diseñados para llevar a cabo este análisis fueron los Escenarios 16, 17 y 18. Estos escenarios consideran concentraciones de 1500, 2500 y 2000 ppm, respectivamente. Los resultados para este análisis se encuentran en la Tabla 14 y la Gráfica 8.

\section{RESULTADOS}

\section{Escenarios optimizados}

Con el fin de seleccionar el esquema adecuado de inyección de baches SP, se consideró optimizar los escenarios con mejor producción de petróleo, a partir de una variación en la tasa de inyección. Esto se hace en procesos de re- cobro mejorado documentados (Sheng, 2013). Una tasa de inyección inferior garantiza un mayor tiempo de producción de petróleo, y una menor degradación mecánica del polímero, asegurando una mayor recuperación del mismo. Por esta razón la tasa de inyección de los Escenarios 14 y 18 se cambió a 600 BFPD, comparados con el caso base. Dichos escenarios son: Escenario 19 y Escenario 20.

- Escenario \#19. Este escenario está diseñado con base en el Escenario \#18, considerando las mismas concentraciones de los químicos (2000 ppm para el surfactante y el polímero), las mismas fechas de inyección y la misma distribución de químicos (porcentaje del volumen poroso del arreglo), la diferencia radica en la disminución de la tasa de inyección (Qiny: 600 BFPD). Las condiciones de inyección de este escenario se encuentran en la Tabla 15.

- Escenario \#20. Este escenario está diseñado a partir del escenario 14. El cual considera una concentración de surfactante equivalente a 3500 ppm y una concentración de polímero de 2500 ppm. Algunas de las condiciones operativas de este escenario se encuentran explícitas en la Tabla 16. Las fechas de inicio y fin de inyección programadas para este escenario, corresponden a las mismas del Escenario 19.

Los resultados obtenidos, a partir de la evaluación a escala de simulación de los Escenarios 19 y 20, se indican en la Gráfica 9 y la Tabla 17.

Resultados comparativos con otras tecnologias

Como se indicó con antelación, otros métodos de recobro fueron evaluados (Inyección de agua, inyección de polímeros e inyección SP convencional). Dado que el Escenario 20 fue el que arrojó mejores resultados, en cuanto a producción de petróleo incremental, éste se usó para la comparación de resultados. La producción de 
petróleo para el Escenario 20 alcanzó los 3.1268 MMbbl, considerando la inyección SP convencional, esta fue de $3.0093 \mathrm{MMbbl}$ al final del periodo de evaluación, resultando en un incremento -a partir de los resultados obtenidos bajo el esquema de inyección de agua- del $14.57 \%$ y del $10.27 \%$, respectivamente. Los resultados de este comparativo se encuentran descritos en la Gráfica 10 y la Tabla 18.

Por otra parte, entre los dos mejores escenarios desde el punto de vista técnico (Escenario \# 19 y Escenario \# 20) el escenario \# 20 fue el que mostró mayor rentabilidad, pues registra el VPN más alto (USD 2,090,835.98). De la misma manera, la TIR y la relación beneficio-costo fueron las más altas; 42.70 por ciento y 1.77 dólares recibidos por dólar invertido, respectivamente.

Para un mayor detalle del análisis financiero se recomienda revisar las variables consideradas para su elaboración (Tabla 19) y los resultados obtenidos para todos los escenarios propuestos a lo largo de este documento (Tabla 20).

\section{CONCLUSIONES}

A partir del análisis y evaluación técnica de la inyección de baches surfactante/polímero, se ha demostrado la viabilidad técnica de esta tecnología, como método de recobro químico aplicable al Campo Yariguí-Cantagallo. Se representa en la mayor recuperación de petróleo y la menor producción de agua (Escenario 20). Aunado al hecho, el mayor factor de recobro obtenido, comparado con las tecnologías de recobro secundario, y de recobro terciario, evaluados en este proyecto.

La inyección de baches SP demostró obtener un aumento del factor de recobro, comparado con la inyección de agua, inyección de polímeros $e$ inyección convencional SP. Esto indica que el surfactante como agente tenso activo, logro invadir el yacimiento a través de su complejidad geológica, reduciendo la tensión interfacial (IFT), y generando una disminución en la saturación de petróleo residual.

Basados en los resultados obtenidos, dos baches son suficientes para obtener una máxima recuperación de petróleo. Por lo tanto, el número de baches no afecta directamente el incremento en la recuperación de petróleo. Esto puede estar asociado a una ruptura temprana del frente de invasión, y a la alta heterogeneidad del yacimiento.

La concentración de los químicos resultó ser un factor fundamental de diseño de los baches $\mathrm{SP}$, implementados en esta evaluación técnica. La mejor recuperación de petróleo fue alcanzada cuando las concentraciones, tanto de polímero como de surfactante, fueron las más altas. Sin embargo, dicha recuperación requirió de la aplicación de una mayor cantidad de químicos, lo que resultó afectando el retorno de la inversión (mayor tiempo de recuperación de la inversión) y ganancia de capital en dicho escenario.

A medida que aumenta el porcentaje de surfactante en el bache (Volumen poroso ocupado por el surfactante) se obtiene una menor recuperación de petróleo. Esto se encuentra asociado a una perdida en el control de movilidad, y a la alta heterogeneidad del yacimiento, lo que facilita la canalización en el yacimiento de los fluidos inyectados (solución tensoactiva y agua).

En el diseño de las tasas de inyección, se evidenció que la tasa óptima es 1500 BFPD. Sin embargo, se encontró que, al disminuir la tasa de inyección (hasta 600 BFPD), se genera una mayor recuperación. Esto está asociado a que los químicos, tienen un mayor tiempo de interactuar con el sistema roca-fluido.

El diseño de baches más pequeños y en mayor número, no genera una mayor recuperación de petróleo, puesto que el efecto de disminución de tensión interfacial asociada, no se desarrolla en gran medida. Esto resulta en la fuerte disminución de la acción del surfactante. 


\section{Agradecimientos}

Los autores expresan su agradecimiento al Ecopetrol S.A. que mediante el Instituto Colombiano del Petróleo, suministró la información para el desarrollo de este artículo. Al Ingeniero Robinson Jiménez por su constante interés en el desarrollo del proyecto y a la Universidad de América por brindar las herramientas requeridas para construir los modelos de simulación.

\section{REFERENCIAS}

Aldana, H.A., Gutiérrez, C.A., Ramos, J.L., Jiménez, R. Estudio de la implementación de esquemas adecuados de inyección de baches surfactante/polímero para el Campo Yariguí-Cantagallo operado por Ecopetrol S.A. mediante simulación numérica.

Rao, D., Ayirata, S., Abe, A. \& Xu, W. (2006) "Impact of low-cost dilute surfactants on wettability and relative permeability". Spe, louisiana state U. (SPE 99609) 


\section{ANEXOS}

Tabla 1. Propiedades del polímero.

\begin{tabular}{|l|l|l|}
\hline \multicolumn{1}{|c|}{ Propiedad } & \multicolumn{1}{c|}{ Valor } & \multicolumn{1}{c|}{ Unidades } \\
\hline Peso molecular del polímero & 8000 & $\mathrm{Lb} / \mathrm{lb}-\mathrm{mol}$ \\
\hline Densidad del polímero & 63.1621 & $\mathrm{Lb} / \mathrm{ft}^{3}$ \\
\hline Viscosidad del polímero & 81 & $\mathrm{cP}$ \\
\hline Adsorción máxima de la roca & $8.271707 \mathrm{E}-06$ & $\mathrm{lb}-\mathrm{mol} / \mathrm{ft}^{3}$ \\
\hline Adsorción residual de la roca & $4.135854 \mathrm{E}-06$ & $\mathrm{lb}-\mathrm{mol} / \mathrm{ft}^{3}$ \\
\hline Volumen poroso accesible & 0.7 & Adimensional \\
\hline Factor de resistencia residual & 2.82 & Adimensional \\
\hline
\end{tabular}

Tabla 2. Propiedades del surfactante.

\begin{tabular}{|l|l|l|}
\hline \multicolumn{1}{|c|}{ Propiedad } & \multicolumn{2}{c|}{ Ualor } \\
\hline Grupo OE/Alcohol & $\mathrm{C}_{9} \mathrm{C}_{11}$ & $\mathrm{lb} / \mathrm{lb}-\mathrm{mol}$ \\
\hline Peso molecular del surfactante & 527 & $\mathrm{lb} / \mathrm{tt}^{3}$ \\
\hline Densidad del surfactante a $104^{\circ} \mathrm{F}$ & 62.18 & $\mathrm{cP}$ \\
\hline Viscosidad del surfactante a $104^{\circ} \mathrm{F}$ & 30 & $\mathrm{lb}-\mathrm{mol} / \mathrm{ft}^{3}$ \\
\hline Adsorción máxima de la roca & $2.524434 \mathrm{E}-03$ & $\mathrm{lb}-\mathrm{mol} / \mathrm{ft}^{3}$ \\
\hline Adsorción residual de la roca & $1.262217 \mathrm{E}-03$ & Adimensional \\
\hline $\mathrm{pH}$ & $6.0-7.5$ & $\mathrm{ppm}$ \\
\hline $\mathrm{CMC}$ en solución acuosa & 270 & \\
\hline
\end{tabular}

Fuente: SPE 99609-MS-P. Impact of Low-Cost Dilute Surfactants on Wettability and Relative Permeability, 2000, p.

Tabla 3. Resultados de las pruebas de núcleos.

\begin{tabular}{|l|c|c|c|c|c|}
\hline $\begin{array}{l}\text { Concentración del } \\
\text { surfactante (ppm) }\end{array}$ & Swi & Sor & Kro @ Swirr & $\begin{array}{c}\text { Krw @ Sw } \\
\text { max }\end{array}$ & $\begin{array}{c}\text { Tensión interfacial } \\
\text { (dina/cm) }\end{array}$ \\
\hline 0 & 0.400 & 0.290 & 0.970 & 0.0710 & 23.37 \\
\hline 500 & 0.500 & 0.210 & 0.830 & 0.1435 & 7.79 \\
\hline 1500 & 0.520 & 0.085 & 0.945 & 0.0959 & 3.1 \\
\hline 3500 & 0.650 & 0.038 & 1.000 & 0.1322 & 0.13 \\
\hline
\end{tabular}

Fuente: SPE 99609-MS-P. Impact of Low-Cost Dilute Surfactants on Wettability and Relative Permeability, 2000, p. 10. Modificado por los autores. 
LINNEA DE INVESTIGACIÓN: SIMULACIÓN DE YACIMIENTOS

Tabla 4. Datos base para el diseño de los modelos de ajuste.

\begin{tabular}{|c|c|c|c|}
\hline Swirr & Sor & Kro @ Swirr & Krw @ Swmax \\
\hline 0.46 & 0.15 & 1 & 0.06 \\
\hline
\end{tabular}

Tabla 5. Efecto del surfactante sobre los fluidos del yacimiento.

\begin{tabular}{|c|c|c|c|c|c|}
\hline Cs (ppm) & Swi & Sor & Kro & Krw & IFT (dina/cm) \\
\hline 0 & 0.4600 & 0.1500 & 1.000 & 0.0592 & 23.37 \\
\hline 500 & 0.5427 & 0.1086 & 1.000 & 0.0675 & 7.79 \\
\hline 1500 & 0.6208 & 0.0440 & 1.000 & 0.0842 & 3.1 \\
\hline 3500 & 0.7285 & 0.0197 & 1.000 & 0.1176 & 0.13 \\
\hline
\end{tabular}

Tabla 6. Escenario \#1 de inyección de baches surfactante/polímero

\begin{tabular}{|c|c|}
\hline \multicolumn{2}{|c|}{ Escenario \# 1 (Qiny: 1500 BFPD) } \\
\hline Fecha & Porcentaje del Volumen Poroso del arreglo \\
\hline 01/01/2015 & Surfactante: $0.5 \%$ \\
\hline $12 / 03 / 15$ & Polímero: $4.5 \%$ \\
\hline $06 / 12 / 20^{\prime} 16$ & Surfactante: $0.5 \%$ \\
\hline $15 / 02 / 17$ & Polímero: $4.5 \%$ \\
\hline $12 / 11 / 2018$ & Agua \\
\hline
\end{tabular}

Gráfica 11. Incidencia del número de baches en la recuperación de petróleo y agua.

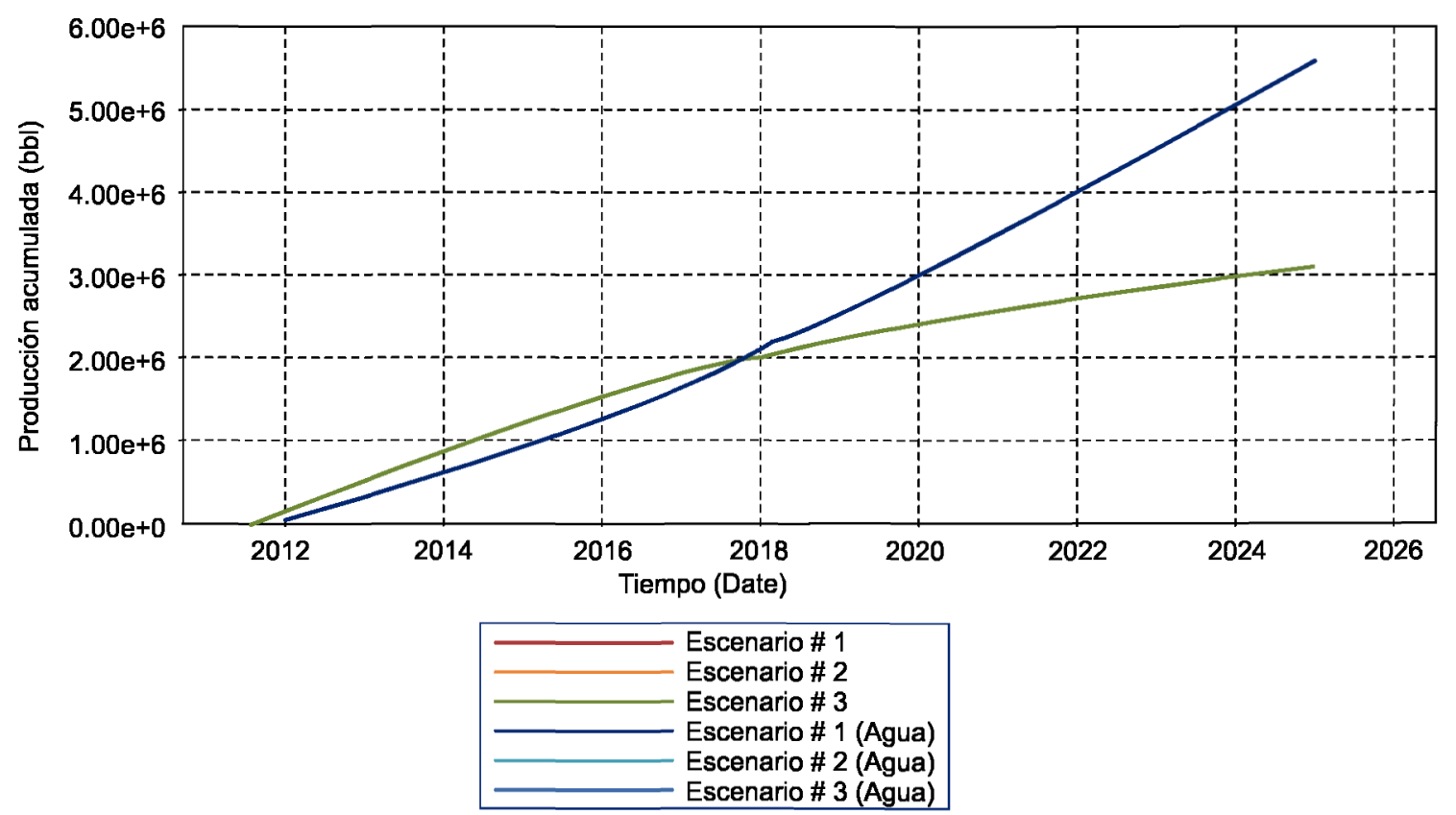


Tabla 72. Incidencia del número de baches en la recuperación de petróleo y agua.

\begin{tabular}{|c|c|c|}
\hline \multicolumn{3}{|c|}{ Número de Baches SP } \\
\hline Escenario & Np (bbl) & Wp (b bl) \\
\hline Escenario \# 1 & $3^{\prime} 101,720$ & $5^{\prime} 556,120$ \\
\hline Escenario \# 2 & $3^{\prime} 099,470$ & $5^{\prime} 558,420$ \\
\hline Escenario \# 3 & $3^{\prime} 085,340$ & $5^{\prime} 575,510$ \\
\hline
\end{tabular}

Gráfica 23. Incidencia de la distribución de los baches, en la recuperación de petróleo y agua.

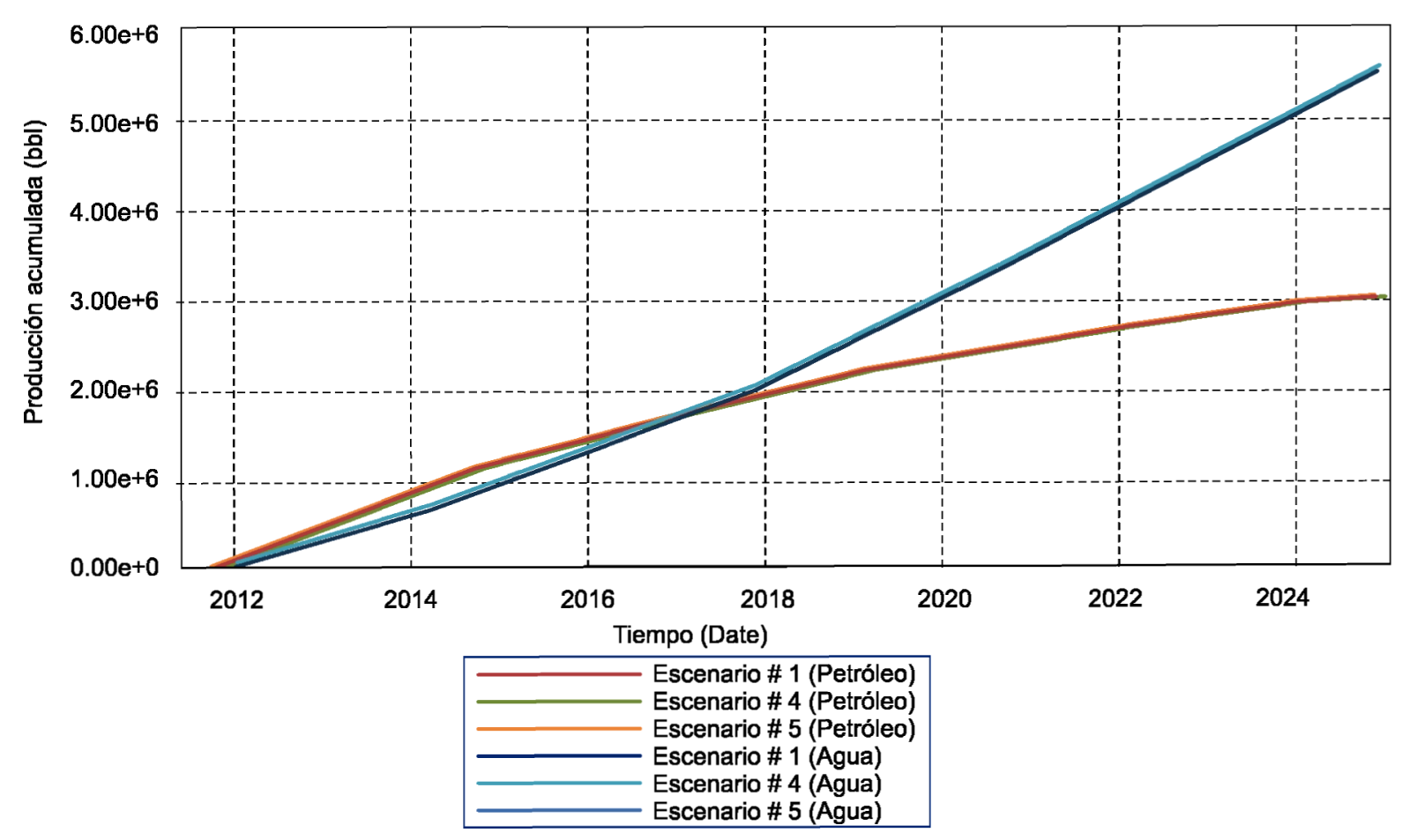

Tabla 84. Incidencia de la distribución de los baches, en la recuperación de petróleo y agua.

\begin{tabular}{|c|c|c|}
\hline \multicolumn{3}{|c|}{ Número de Baches SP } \\
\hline Escenario & Np (bbl) & $W_{p}(\mathrm{bbl})$ \\
\hline Escenario \# 1 & $3^{\prime} 101,720$ & $5^{\prime} 556,120$ \\
\hline Escenario \# 4 & $3^{\prime} 036,710$ & $5^{\prime} 620,990$ \\
\hline Escenario \# 5 & $3^{\prime} 072,270$ & $5^{\prime} 585,590$ \\
\hline
\end{tabular}


Gráfica 31. Incidencia en la recuperación de petróleo y agua, en función de la tasa de inyección.

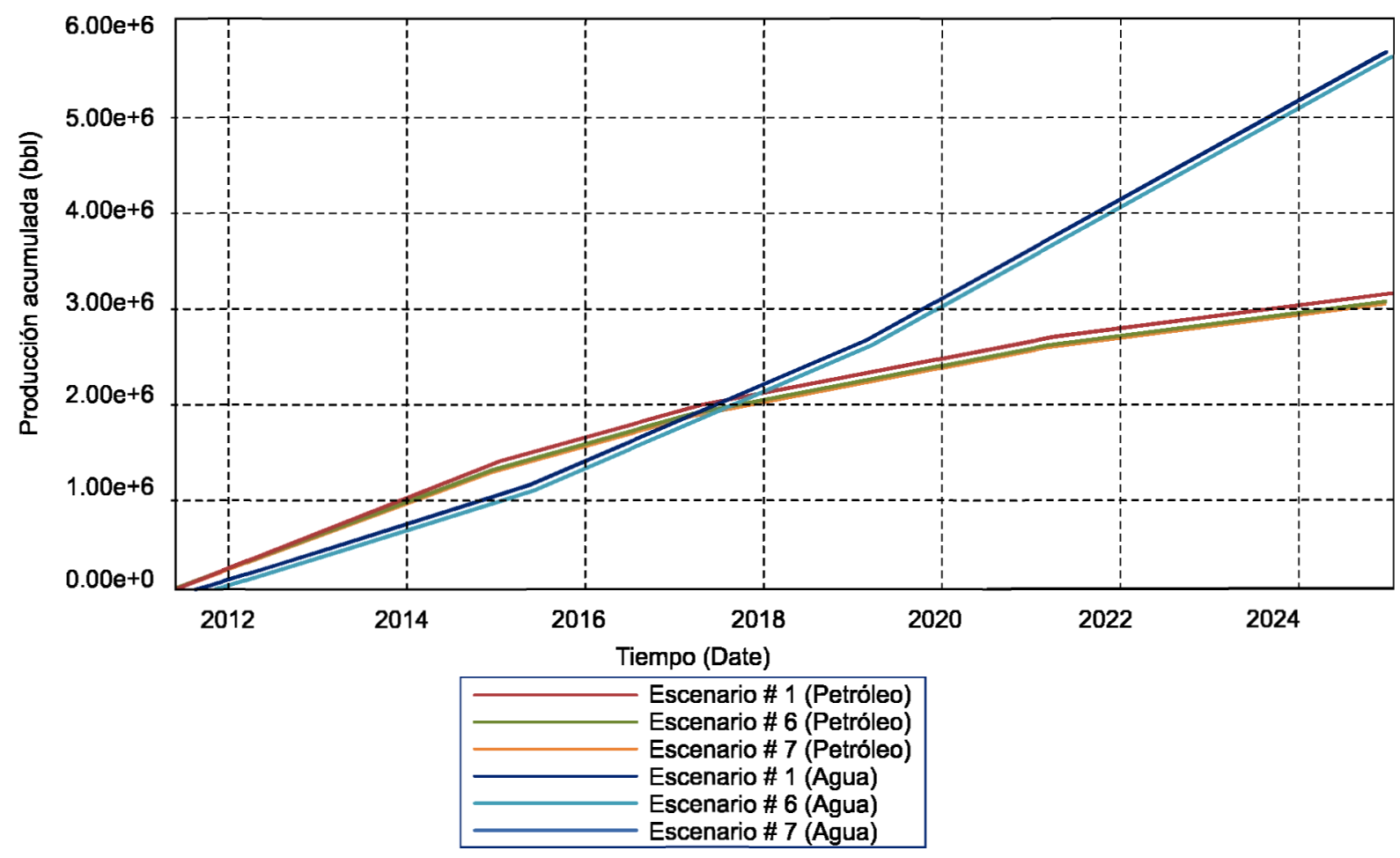

Tabla 9. Incidencia de la tasa de inyección, en la recuperación de petróleo y agua.

\begin{tabular}{|c|c|c|}
\hline \multicolumn{3}{|c|}{ Tasa de inyección } \\
\hline Escenario & Np (bbl) & Wp (b bl) \\
\hline Escenario \# 1 & $3^{\prime} 101,720$ & $5^{\prime} 556,120$ \\
\hline Escenario \# 6 & $3^{\prime} 080,910$ & $5^{\prime} 576,840$ \\
\hline Escenario \# 7 & $3^{\prime} 013,780$ & $5^{\prime} 644,000$ \\
\hline
\end{tabular}


Gráfica 4. Incidencia en la recuperación de petróleo y agua, en función del tamaño de los baches.

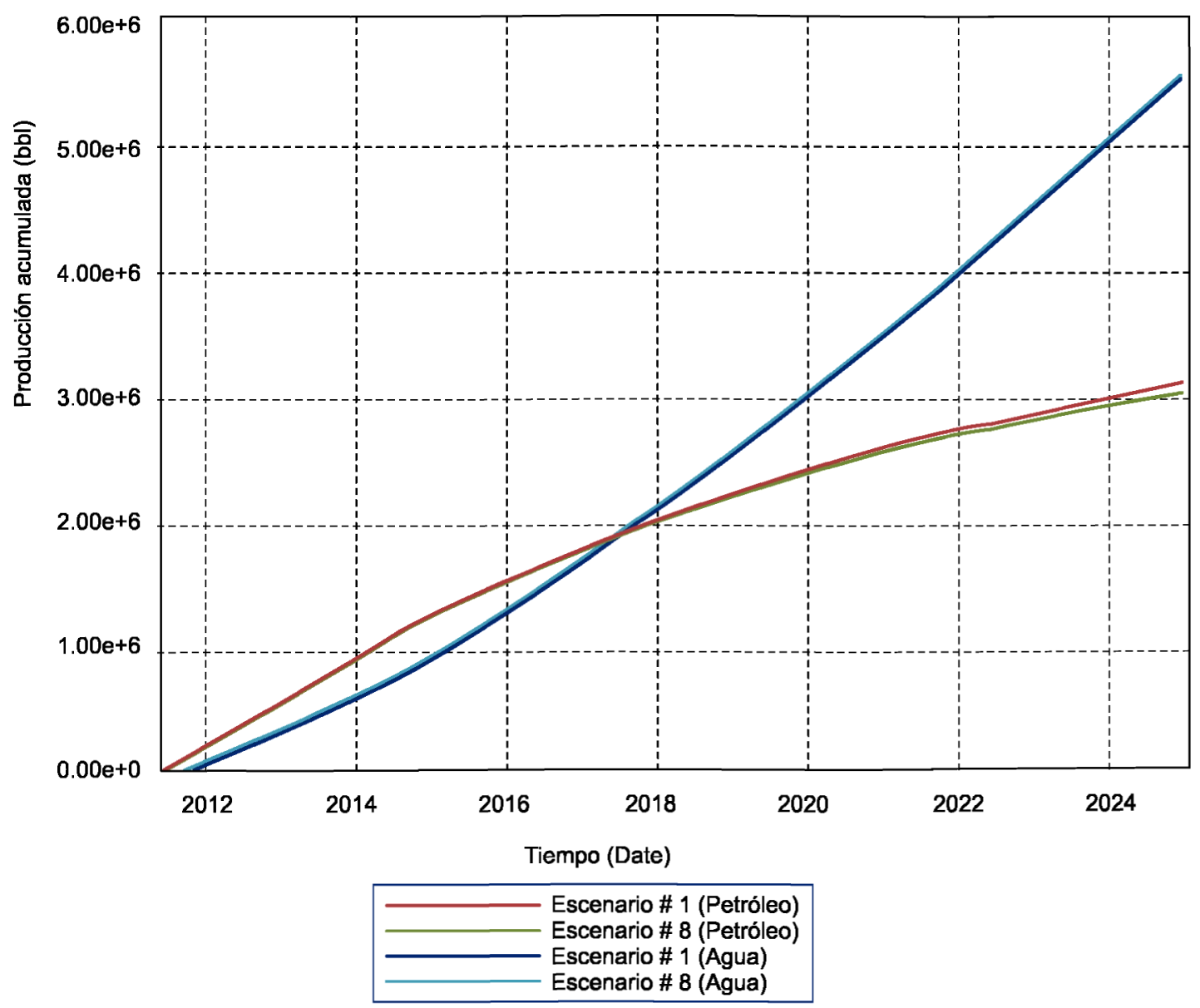

Tabla 10. Incidencia del tamaño de los baches, en la recuperación de petróleo y agua.

\begin{tabular}{|r|c|c|}
\hline \multicolumn{3}{|c|}{ Tamaño de los bache } \\
\hline Escenario & Np (bbl) & Wp (bbl) \\
\hline Escenario \# 1 & 3'101,720 & $5^{\prime} 556,120$ \\
\hline Escenario \# 8 & $3^{\prime} 060,850$ & $5^{\prime} 587,830$ \\
\hline
\end{tabular}


Gráfica 5 .Incidencia de la concentración del surfactante, en la recuperación de petróleo y agua.

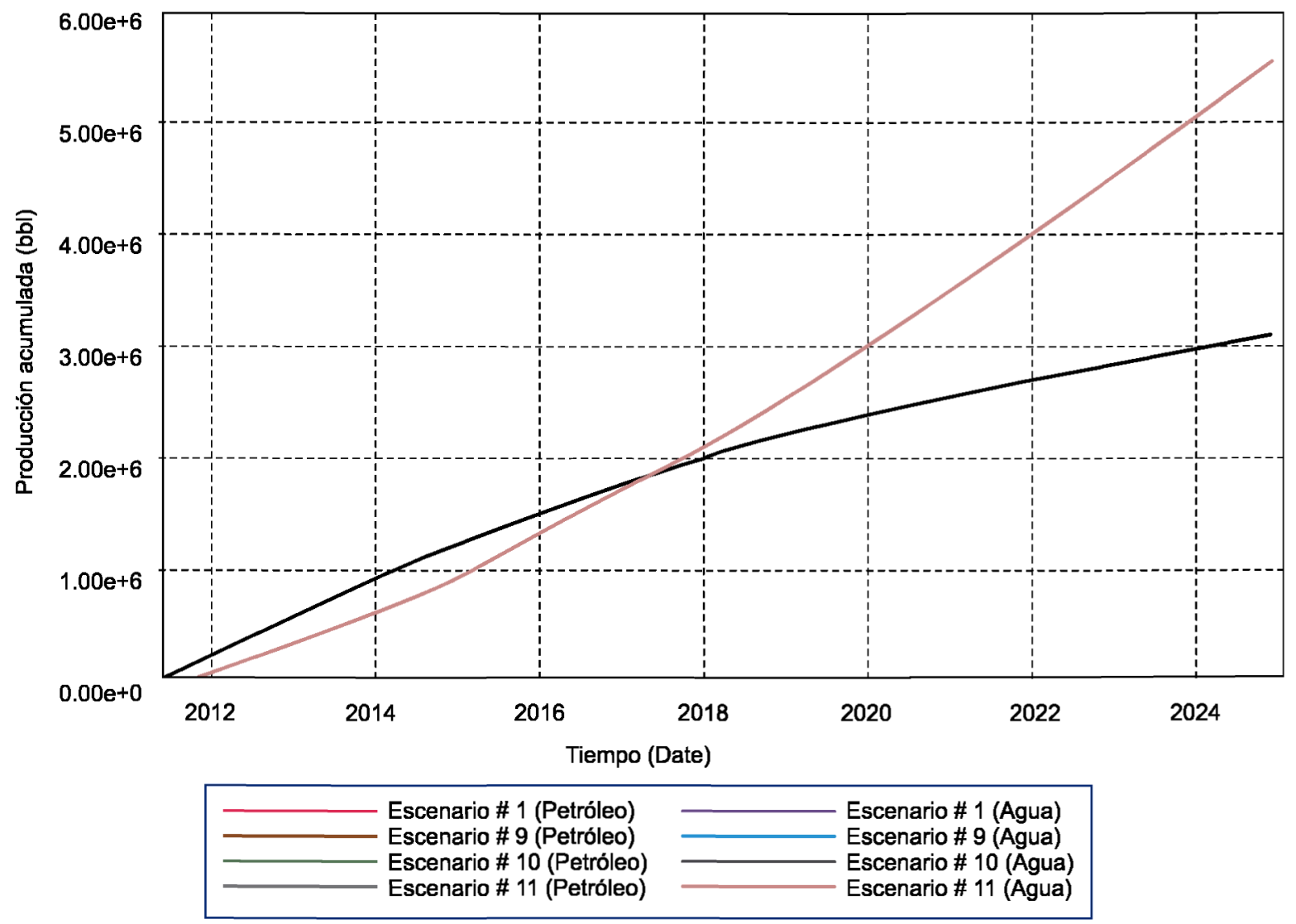

Tabla 511 Incidencia de la concentración del surfactante, en la recuperación de petróleo y agua.

\begin{tabular}{|c|c|c|}
\hline \multicolumn{3}{|c|}{ Concentración del surfactante } \\
\hline Escenario & Np (bbl) & Wp (bbl) \\
\hline Escenario \# 1 & $3^{\prime} 101,720$ & $5^{\prime} 556,120$ \\
\hline Escenario \# 9 & $3^{\prime} 079,950$ & $5^{\prime} 577,940$ \\
\hline Escenario \# 10 & $3^{\prime} 095,060$ & $5^{\prime} 562,790$ \\
\hline Escenario \# 11 & $3^{\prime} 072,160$ & $5^{\prime} 585,730$ \\
\hline
\end{tabular}


Gráfica 66. Incidencia de la concentración del polímero, en la recuperación de petróleo y agua.

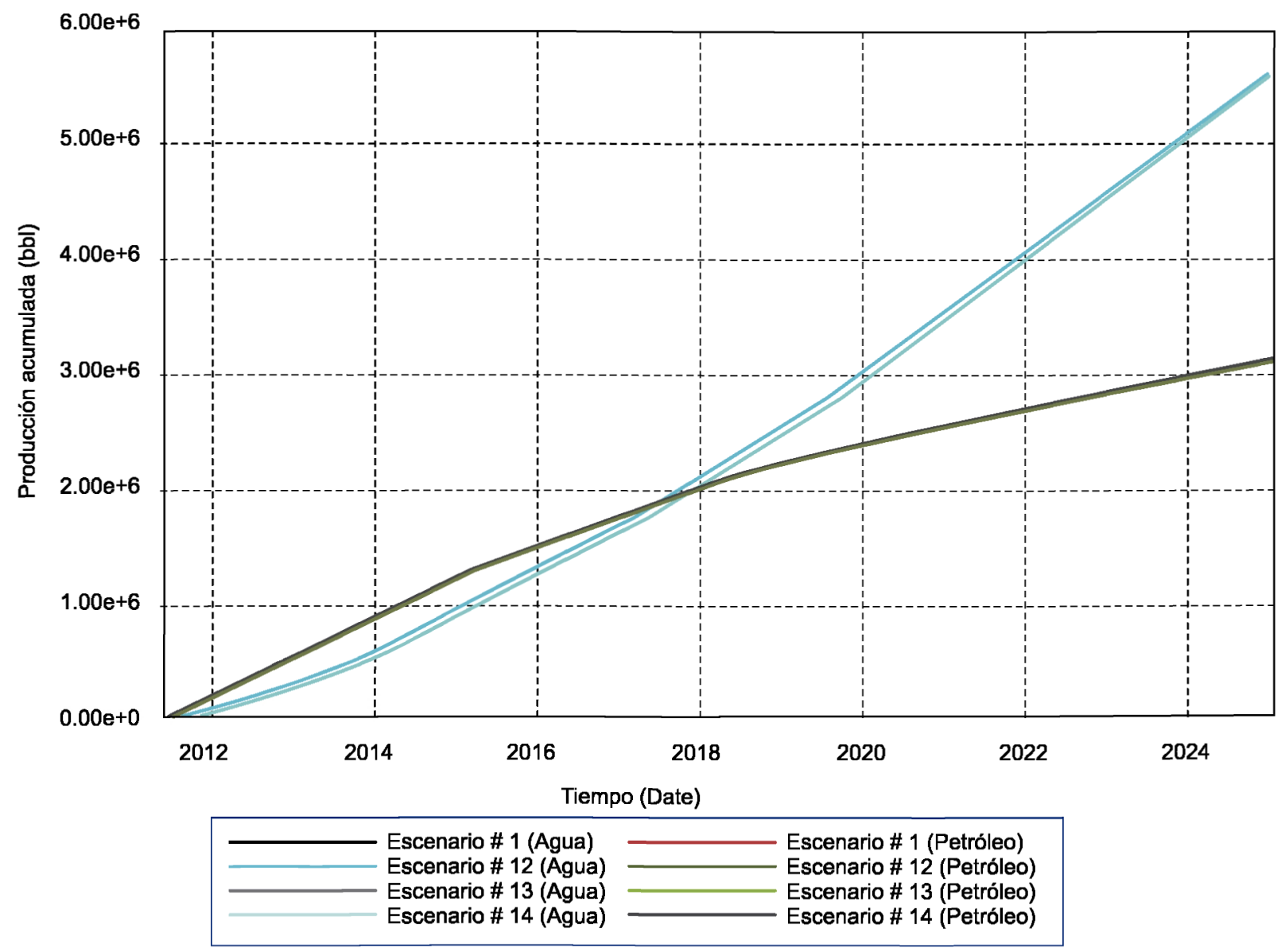

Tabla 127. Incidencia de la concentración del polímero, en la recuperación de petróleo y agua

\begin{tabular}{|c|c|c|}
\hline \multicolumn{3}{|c|}{ Concentración del polímero } \\
\hline Escenario & $N_{p}$ (bbl) & $W_{p}(\mathrm{~b} b \mathrm{bl})$ \\
\hline Escenario \# 1 & $3^{\prime} 101,720$ & $5^{\prime} 556,120$ \\
\hline Escenario \# 12 & $3^{\prime} 065,110$ & $5^{\prime} 592,620$ \\
\hline Escenario \# 13 & $3^{\prime} 107,170$ & $5^{\prime} 550,690$ \\
\hline Escenario \# 14 & $3^{\prime} 118,070$ & $5^{\prime} 539,810$ \\
\hline
\end{tabular}


Gráfica 7. Incidencia del tamaño de los baches, en la recuperación de petróleo y agua.

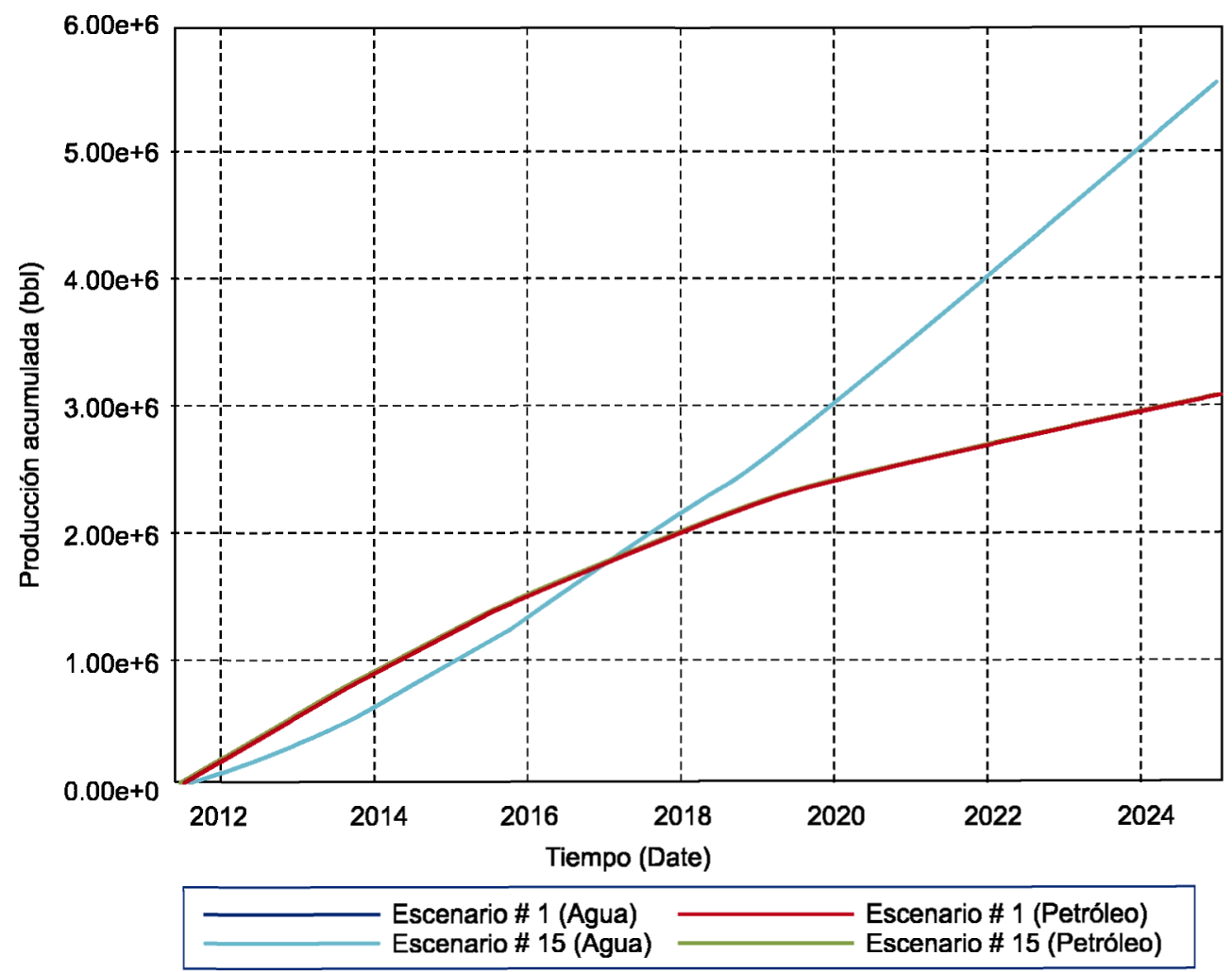

Tabla 13. Incidencia del tamaño de los baches, en la recuperación de petróleo y agua.

\begin{tabular}{|c|c|c|}
\hline \multicolumn{3}{|c|}{ Tamaño de los bache } \\
\hline Escenario & Np (bbl) & Wp (bbl) \\
\hline Escenario \# 1 & $3^{\prime} 101,720$ & $5^{\prime} 556,120$ \\
\hline Escenario \# 15 & $3^{\prime} 089,710$ & $5^{\prime} 568,130$ \\
\hline
\end{tabular}

Tabla 148. Incidencia de concentraciones de inyección iguales, en la recuperación de petróleo y agua.

\begin{tabular}{|c|c|c|}
\hline \multicolumn{3}{|c|}{ Concentraciones de inyección iguales } \\
\hline Escenario & Np (bbl) & Wp (bbl) \\
\hline Escenario \# 1 & $3^{\prime} 101,720$ & $5^{\prime} 556,120$ \\
\hline Escenario \# 16 & $3^{\prime} 100,610$ & $5^{\prime} 557,260$ \\
\hline Escenario \# 17 & $3^{\prime} 105,170$ & $5^{\prime} 552,710$ \\
\hline Escenario \# 18 & $3^{\prime} 119,420$ & $5^{\prime} 538,450$ \\
\hline
\end{tabular}


Tabla 15. Escenario \#19 de inyección de baches surfactante/polímero

\begin{tabular}{|l|l|}
\hline \multicolumn{2}{|c|}{ Escenario \# 19 (Qiny: 600 BFPD) } \\
\hline \multicolumn{2}{|c|}{ Cs = 2000 ppm; Cp = 2000 ppm } \\
\hline Fecha & \multicolumn{1}{|c|}{ Porcentaje del Volumen Poroso del arreglo } \\
\hline $01 / 01 / 2015$ & Surfactante: $0.5 \%$ \\
\hline $26 / 06 / 2015$ & Polímero: $4.5 \%$ \\
\hline $31 / 10 / 2019$ & Surfactante: $0.5 \%$ \\
\hline $25 / 04 / 2020$ & Polímero: $4.5 \%$ \\
\hline $30 / 08 / 2024$ & Agua \\
\hline
\end{tabular}

Gráfica 8. Incidencia en la recuperación de petróleo y agua, en función de concentraciones de inyección iguales.

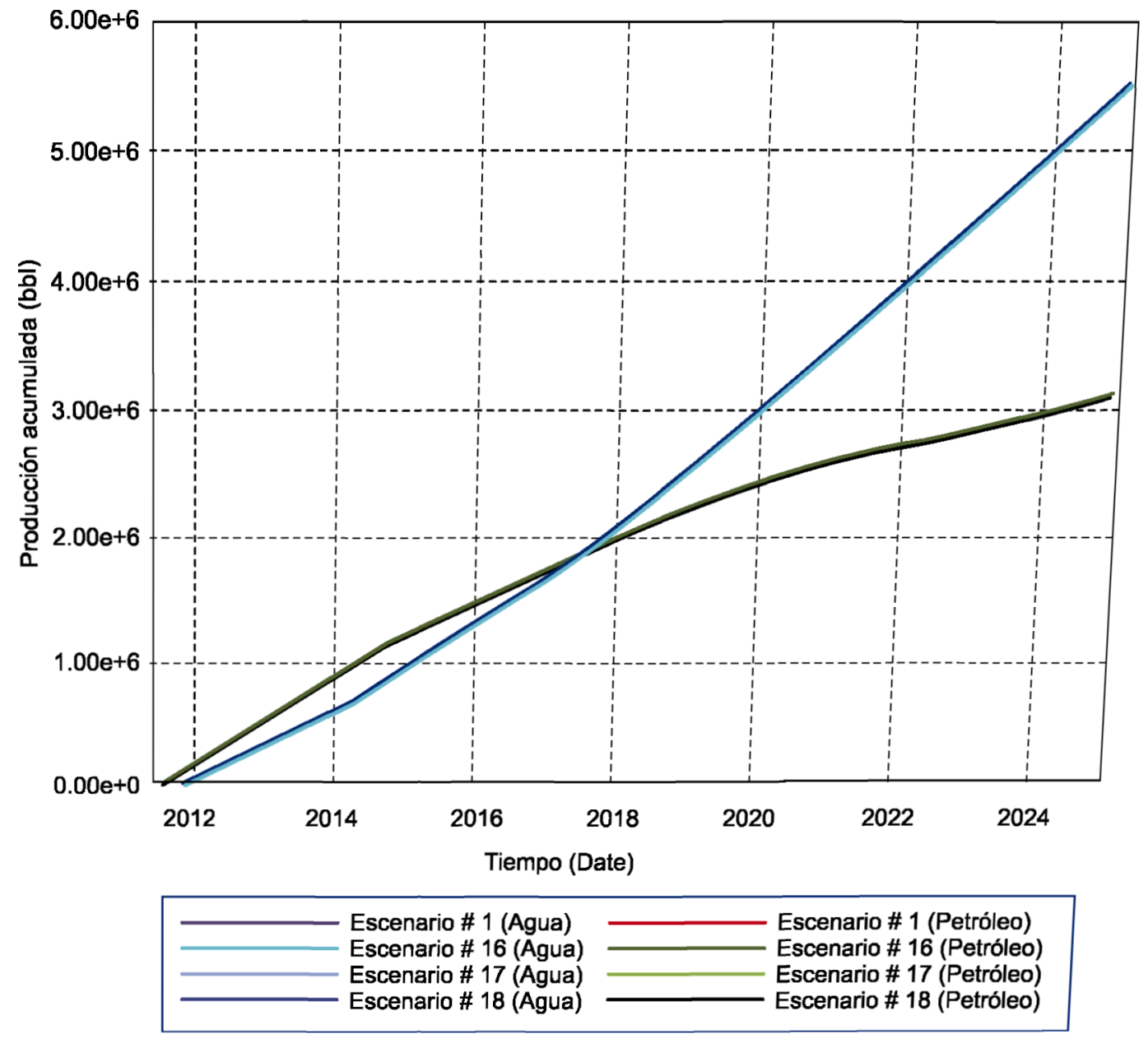


Gráfica 92. Resultados de la recuperación de petróleo y agua, de los Escenarios \#19 y \#20.

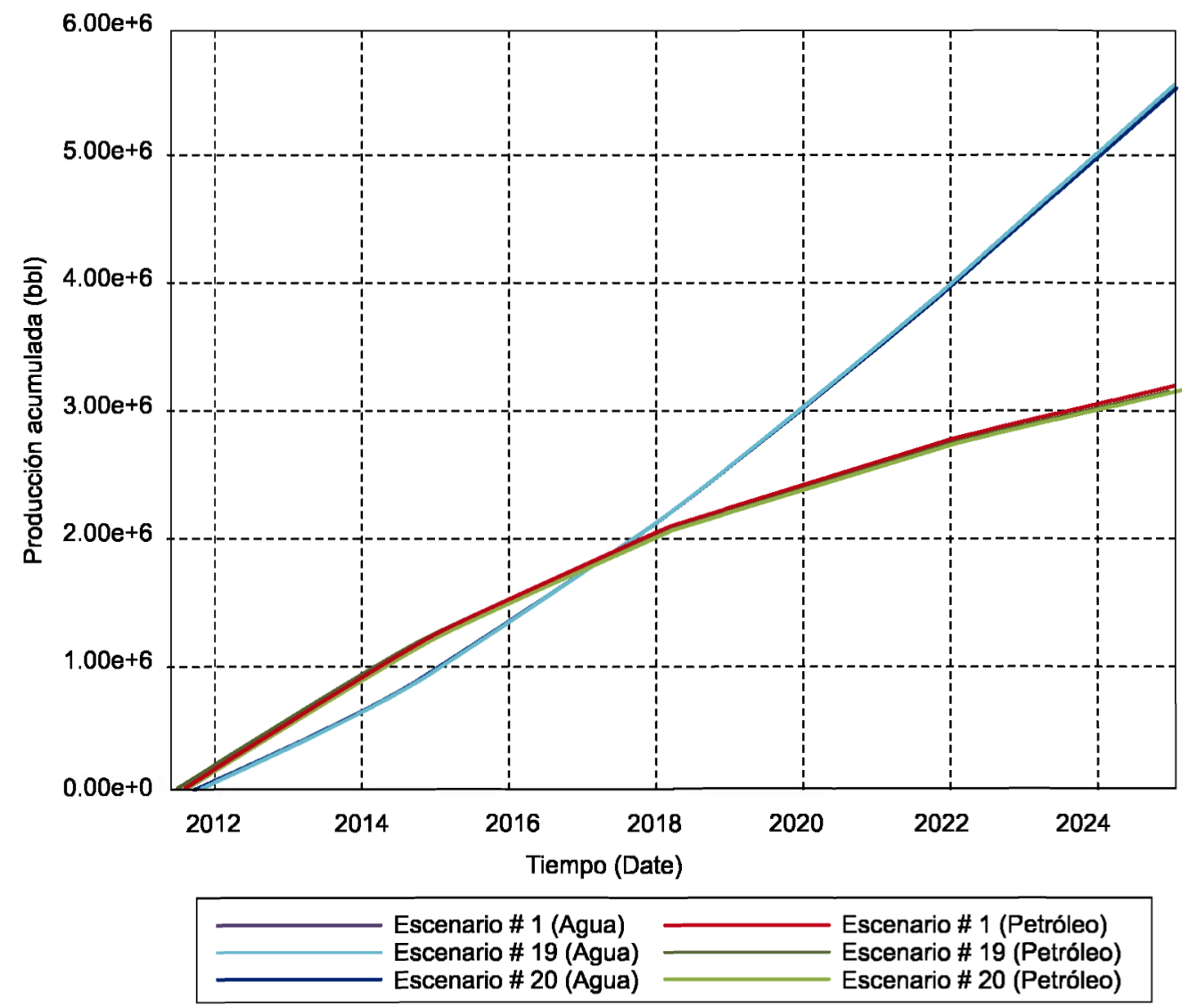

Tabla 169. Escenario \#20 de inyección de baches surfactante/polímero

\begin{tabular}{|l|l|}
\hline \multicolumn{2}{|c|}{ Escenario \# 20 (Qiny: 600 BFPD) } \\
\hline \multicolumn{1}{|c|}{ Cs = 3500 ppm; Cp = 2500 ppm } \\
\hline $01 / 01 / 2015$ & \multicolumn{1}{|c|}{ Porcentaje del Volumen Poroso del arreglo } \\
\hline $26 / 06 / 2015$ & Surfactante: $0.5 \%$ \\
\hline $31 / 10 / 2019$ & Polímero: $4.5 \%$ \\
\hline $25 / 04 / 2020$ & Surfactante: $0.5 \%$ \\
\hline $30 / 08 / 2024$ & Polímero: $4.5 \%$ \\
\hline
\end{tabular}

Tabla 1710. Recuperación de petróleo y agua bajo esquemas optimizados.

\begin{tabular}{|l|c|c|}
\hline \multicolumn{3}{|c|}{ Escenarios óptimos } \\
\hline Escenario & Np (bbl) & Wp (bbl) \\
\hline Escenario \# 1 & $3^{\prime} 101,720$ & $5^{\prime} 556,120$ \\
\hline Escenario \# 19 & $3^{\prime} 122,640$ & $5^{\prime} 535,180$ \\
\hline Escenario \# 20 & $3^{\prime} 126,840$ & $5^{\prime} 531,020$ \\
\hline
\end{tabular}


Gráfica 10 3. Producción acumulada de petróleo por cada tecnología evaluada

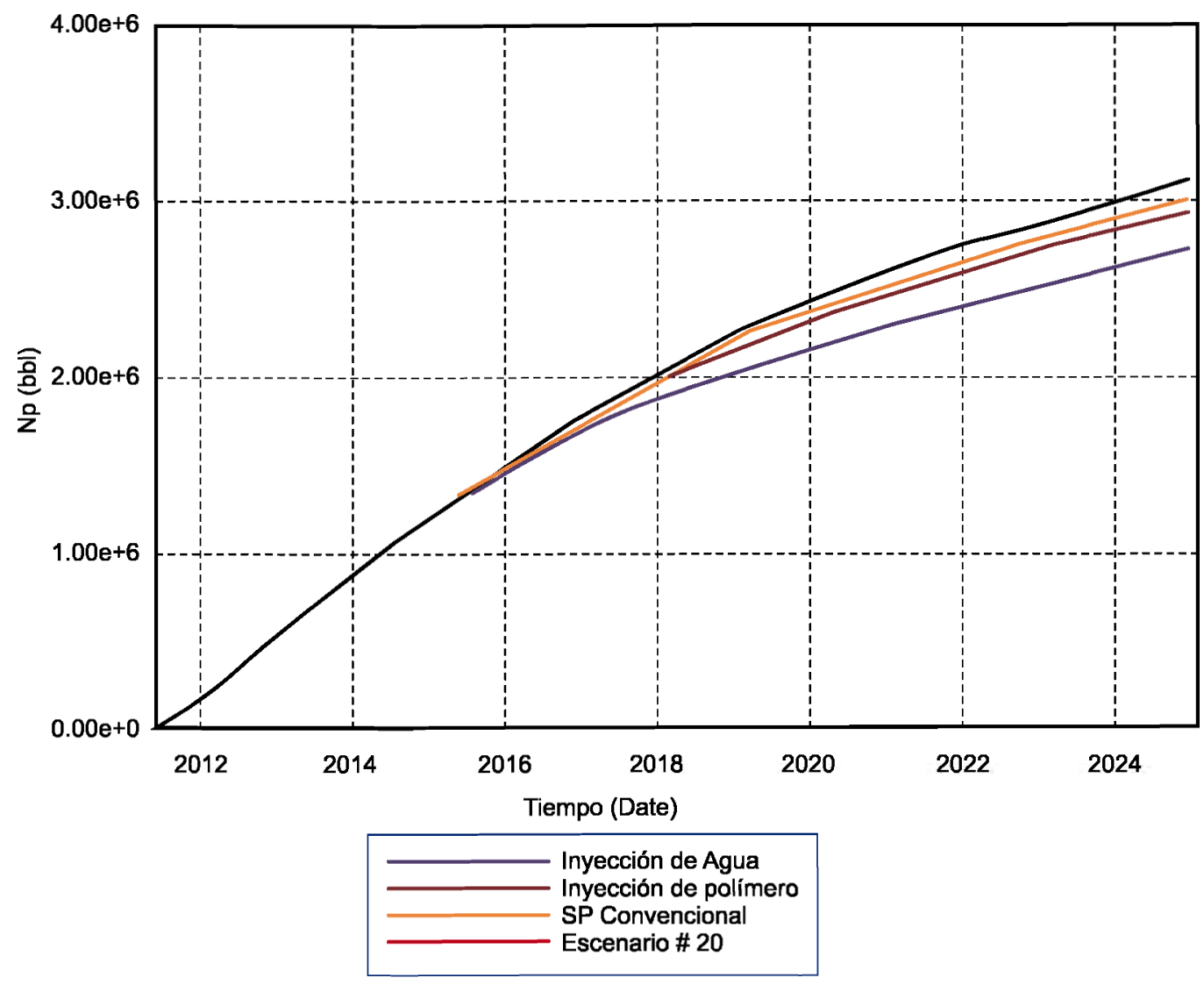

Tabla 18. Producción acumulada de petróleo y agua por cada tecnología evaluada.

\begin{tabular}{|l|l|l|}
\hline \multicolumn{3}{|c|}{ Baches SP } \\
\hline Escenario & \multicolumn{1}{|c|}{ Np } & Wp \\
\hline Agua & 2'729,090 & $5^{\prime} 928,370$ \\
\hline Polímero & $2^{\prime} 947,890$ & $5^{\prime} 710,360$ \\
\hline SP Conv. & $3^{\prime} 009,280$ & $5^{\prime} 648,160$ \\
\hline Escenario \# 20 & $3^{\prime} 126,840$ & $5^{\prime} 531,020$ \\
\hline
\end{tabular}

Tabla 19. Condiciones para el análisis financiero.

\begin{tabular}{|l|l|}
\hline \multicolumn{2}{|c|}{ Condiciones financieras } \\
\hline Tasa interna de oportunidad (TIO) & $11.11 \%$ \\
\hline Precio del crudo & USD 47.80 \\
\hline Regalías por producción incremental & $8.71 \%$ \\
\hline Impuestos & $33 \%$ \\
\hline Precio del polímero & USD 2 por libra \\
\hline Precio del surfactante & USD 1.85 por libra \\
\hline Costo de tratamiento de agua & USD 0.5 por barril \\
\hline
\end{tabular}


LÍNEA DE INVESTIGACIÓN: SIMULACIÓN DE YACIMIENTOS

Tabla 20. Indicadores de viabilidad para los escenarios evaluados.

\begin{tabular}{|l|c|}
\hline \multicolumn{1}{|c|}{ Escenario } & VPN \\
\hline Iny. De Polímeros & $\$ 780,905.64$ \\
\hline Iny. SP Conv. & $\$ 1,032,550.31$ \\
\hline \multicolumn{1}{|c|}{ Baches SP } & VPN \\
\hline Escenario \#1 & $\$ 1,613,414.43$ \\
\hline Escenario \#2 & $\$ 1,106,207.97$ \\
\hline Escenario \#3 & $\$(29,122.47)$ \\
\hline Escenario \#4 & $\$ 982,436.83$ \\
\hline Escenario \#5 & $\$ 1,294,129.25$ \\
\hline Escenario \#6 & $\$ 767,773.01$ \\
\hline Escenario \#7 & $\$ 1,344,313.98$ \\
\hline Escenario \#8 & $\$ 1,555,456.44$ \\
\hline Escenario \#9 & $\$ 1,653,278.04$ \\
\hline Escenario \#10 & $\$ 1,368,232.13$ \\
\hline Escenario \#11 & $\$ 1,564,465.26$ \\
\hline Escenario \#12 & $\$ 1,340,371.77$ \\
\hline Escenario \#13 & $\$ 1,107,695.05$ \\
\hline Escenario \#14 & $\$ 1,706,832.84$ \\
\hline Escenario \#15 & $\$ 1,816,310.33$ \\
\hline Escenario \#16 & $\$ 1,217,123.45$ \\
\hline Escenario \#17 & $\$ 1,485,501.59$ \\
\hline Escenario \#18 & $\$ 2,090,835.98$ \\
\hline Escenario \#19 & $\$ 1,733,196.55$ \\
\hline Escenario \#20 & \\
\hline
\end{tabular}

\begin{tabular}{|c|c|}
\hline TIR & $B / C$ \\
\hline $21.35 \%$ & 1.68 \\
\hline $23.82 \%$ & 1.65 \\
\hline TIR & $\mathrm{B} / \mathrm{C}$ \\
\hline $29.09 \%$ & 1.66 \\
\hline $24.40 \%$ & 1.49 \\
\hline $18.23 \%$ & 1.34 \\
\hline $10.84 \%$ & 1.31 \\
\hline $20.86 \%$ & 1.52 \\
\hline $24.84 \%$ & 1.59 \\
\hline $21.17 \%$ & 1.50 \\
\hline $26.50 \%$ & 1.61 \\
\hline $30.26 \%$ & 1.66 \\
\hline $30.46 \%$ & 1.68 \\
\hline $27.64 \%$ & 1.61 \\
\hline $30.30 \%$ & 1.72 \\
\hline $25.51 \%$ & 1.55 \\
\hline $22.40 \%$ & 1.47 \\
\hline $30.75 \%$ & 1.72 \\
\hline $32.88 \%$ & 1.72 \\
\hline $23.86 \%$ & 1.49 \\
\hline $28.01 \%$ & 1.59 \\
\hline $42.70 \%$ & 1.77 \\
\hline $36.81 \%$ & 1.64 \\
\hline
\end{tabular}

